\title{
Salt-tolerance in Vicia faba L. is mitigated by the capacity of salicylic acid to improve photosynthesis and antioxidant response
}

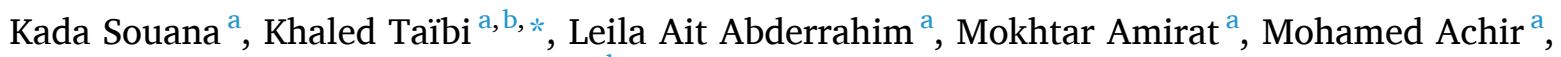 \\ Mohamed Boussaid ${ }^{\mathrm{a}}$, José M. Mulet ${ }^{\mathrm{b}}$ \\ ${ }^{\text {a }}$ Faculty of Life and Natural Sciences, University of Tiaret, 14000, Algeria \\ ${ }^{\mathrm{b}}$ Instituto de Biología Molecular y Celular de Plantas (IBMCP), Universitat Politècnica de València-Consejo Superior de Investigaciones Científicas, Avd. de los Naranjos \\ $s / n$, Valencia, 46022, Spain
}

\section{A R T I C L E I N F O}

\section{Keywords:}

Salt tolerance

salicylic acid

Vicia fabaL.

water status

gas exchanges

photosynthesis

ions homeostasis

antioxidants enzymes

\begin{abstract}
A B S T R A C T
Selection and improvement of crops subjected to salinity constitutes an urgent need for increasing agricultural and food production in order to feed the growing human population. The aim of the present study is to evaluate the role of salicylic acid (SA) application in mitigating the adverse effects of salinity on faba bean (Vicia faba L.) at the physiological and molecular levels. Fort this purpose, two faba bean genotypes were subjected to various concentrations of $\mathrm{NaCl}$ and salicylic acid in a full factorial design. After that, growth, water status, gas exchanges, photosynthesis parameters, ions homeostasis and antioxidant enzymes activities were evaluated. The obtained results demonstrated that salinity induced several limitations in plants growth and physiological attributes. In response, salt stressed faba bean plants improved water status and enhanced antioxidant enzymatic activities. Remarkably, salt-tolerance of both genotypes was significantly improved by salicylic acid application which allowed the maintenance of cell membrane and photosynthetic process, restoring of ion homeostasis and the diminution of oxidative damages. Overall, the difference between genotypes is rather quantitative than qualitative even if the genotype Aguadulce displayed better growth, physiological and molecular response under salt stress than the genotype Histal. Besides, the beneficial effects of salicylic acid vary according to its concentration, the tested genotype and the studied parameter; the genotype Aguadulce performs better under the treatment with $0.5 \mathrm{mM}$ SA while the genotype Histal manifests greater behaviour under the treatment with $1 \mathrm{mM}$ SA. Therefore, salicylic acid can be considered as potential growth regulator to improve the salt response of faba bean. The application of salicylic acid would provide a practical basis for wide cultivation of faba bean in marginal and wastelands under-cultivated and might propose an effective ecological and economical alternative solution to deal with salt-affected soils mainly in arid regions.
\end{abstract}

\section{Introduction}

Salinity is one of the most major threats to plants and crops yields in the XXI century worldwide and its adverse effects are expected to increase under the burden of climate change mainly in arid and semiarid regions. Recent statistics have revealed more than 45 million hectares of irrigated land damaged by salt stress around the world and this number is in perpetual increase (Isayenkov and Maathuis, 2019).

Plants subjected to salinity experience a combination of osmotic and ionic stresses that leads regrettably to various damages at the physiological, cellular and molecular levels, further causing reduced uptake of nutrients and decline of photosynthetic performance (Taïbi et al., 2016).
In addition, salt stress produces plenty of reactive oxygen species which damage the biomolecules (lipids, proteins, and nucleic acids) and other enzymatic activities and even cause the degradation of cell membrane system (Demiral and Türkan, 2005). To deal with this constraint, plants activate several pathways to control salt damage and modulate cellular homeostasis and growth. Salt tolerance is often decrypted by the activation of cell signaling pathways including synthesis of antioxidant enzymes, stress hormones and osmoprotectant metabolites such as amino acids and sugars (Isayenkov and Maathuis, 2019).

Salicylic acid, an endogenous phenolic plant growth regulator, has been found to generate a wide range of metabolic and physiological responses in plants thereby affecting their growth and development

\footnotetext{
* Corresponding author.

E-mail address: khaledtaibi@hotmail.com (K. Taïbi).
} 
(Khan et al., 2015). The positive effects of salicylic acid in mitigating stress are attributed to nutrient uptake improve, membrane protection, photosynthesis repair, as it can interact also with reactive oxygen species signal pathways and reduce oxidative stress (Herrera-Vásquez et al., 2015; Xu and Brosché, 2014). Several reports are available highlighting the role of salicylic acid in improving salt stress tolerance in plants (Ahmad et al., 2018; Jayakannan et al., 2013; Khan et al., 2014; Li et al., 2014a; Li et al., 2014b; Nazar et al., 2015).

Fabaceous plants constitute a suitable solution to feed growing populations, improve human and animal nutrition and enhance soil fertility since they are able to enrich the soil's nitrogen content throughout its symbiotic association with rhizobium (Castro-Guerrero et al., 2016). The faba bean or broad bean, Vicia fabaL., is an important legume for human nutrition as it is a major source of protein, fiber, vitamins and minerals. This species is susceptible to salt regions and attempts are being made to enhance its production and yields mainly in marginal arid lands.

In the present study, we hypothesised that (i) the extent of the beneficial effects of salicylic acid application varies among genotypes belonging to the same species and that (ii) the recovery photosynthetic performance and antioxidant enzymatic activity enhancement under salinity are correlated with the applied concentration of the hormone. Therefore, the main objective of the work is to evaluate the role of salicylic acid application at various concentrations in mitigating the adverse effects of salinity on two faba bean genotypes at the physiological and molecular levels. Examining the magnitude of intra-specific and genotypic variation for salinity tolerance along with a comprehensive description of the physiological and molecular response could provide vital information to faba bean breeding programs for the selection of the most suited genotypes for specific environmental conditions for their introduction in salt affected areas.

\section{Material and methods}

\subsection{Plant material}

Two genotypes of Vicia faba L., Aguadulce and Histal, widely cultivated in developing countries of the Mediterranean region were tested throughout the present study.

\subsection{Growth conditions}

Healthy and homogeneous seeds were surface sterilized using sodium hypochlorite $(0.5 \%, \mathrm{v} / \mathrm{v})$ during 5 minutes followed by several washes with distilled water. After that, seeds were allowed to germinate between wet filter paper in Petri dishes in a growth chamber at controlled conditions $\left(18-25{ }^{\circ} \mathrm{C}\right.$ temperature, $70-90 \%$ relative humidity) in the dark.

Seedlings cultivation were performed as described in Taïbi et al. (2016). Uniform healthy seedlings were selected and individually transplanted in culture pots containing a mixture of commercial peat and vermiculite $(\mathrm{V}: \mathrm{V})$. Culture was conducted under controlled conditions in a greenhouse $\left(600 \mu \mathrm{mol} \mathrm{m}{ }^{-2} \cdot \mathrm{s}^{-1}\right.$ of light intensity, $25 / 18{ }^{\circ} \mathrm{C}$ day/night temperature, $16 \mathrm{~h}$ photoperiod, and $70 \%$ relative humidity). Seedlings were irrigated by a Hoagland full nutrient solution twice to three times a week.

\subsection{Stress application and monitoring}

Experiments were performed on seedlings of four-weeks-old. Salt stress was set-up with $\mathrm{NaCl}$ at two levels of 100 or $200 \mathrm{mM}$. Moreover, the hormone application consisted of treating plants with $0.5 \mathrm{mM}$ or $1.0 \mathrm{mM}$ salicylic acid $\left(\mathrm{C}_{7} \mathrm{H}_{6} \mathrm{O}_{3} ; 2\right.$-hydroxybenzoic acid; Sigma-Aldrich, $\mathrm{C}$ 99.0\%) dissolved in $0.02 \%$ Tween 20 (Polyoxyethylenesorbitan monolaurate, Sigma Chemicals, UK).

Either $\mathrm{NaCl}$ or salicylic acid (mixed with Tween-20) were both dissolved in the nutrient solution of Hoagland which was applied directly to the pots for irrigation.

Control plants were irrigated by Hoagland full nutrient solution without $\mathrm{NaCl}$ and SA. Untreated plants with salicylic acid received the same volume of Tween-20 added to the nutrient solution for homogeneity. It should be noted that no visible damages on the stressed plants were observed during the experiment.

Overall, plants were arranged in a full factorial design with two faba bean genotypes, three salt treatments (i.e. 0, 100 and $200 \mathrm{mM} \mathrm{NaCl}$ ) and three salicylic acid treatments (i.e. $0,0.5$ and $1 \mathrm{mM} \mathrm{SA}$ ). For each treatment, eight replicates were set and processed.

Plants were harvested after ten days of treatment. The dry weights of the aerial parts and roots were determined and samples were conserved at $-80^{\circ} \mathrm{C}$ for further analyses.

\subsection{Water status measurements}

Leaf water potential $\left(\Psi_{\mathrm{w}}, \mathrm{MPa}\right)$ was measured in eight plants selected randomly per treatment using a Scholander-type pressure pump (PMS 1000 , USA). Nevertheless, relative water content (RWC) was assessed on leaves following the formula:

RWC $(\%)=[($ fresh weight-dry weight $) /($ turgid weight-dry weight $)]$ $\mathrm{x} 100$

\subsection{Gas exchange and photosynthesis measurements}

Instantaneous assessments of stomatal conductance $\left(g_{\mathrm{s}}, \mathrm{mol} \mathrm{m}^{-2} \mathrm{~s}^{-1}\right)$, transpiration $\left(E, m m o l \mathrm{H}_{2} \mathrm{O} \mathrm{m} \mathrm{m}^{-2} \mathrm{~s}^{-1}\right)$, net $\mathrm{CO}_{2}$ assimilation $\left(P_{\mathrm{n}}, \mu \mathrm{mol}\right.$ $\left.\mathrm{CO}_{2} \mathrm{~m}^{-2} \mathrm{~s}^{-1}\right)$, intercellular $\mathrm{CO}_{2}$ concentration $\left(\mathrm{Ci}, \mu \mathrm{mol} \mathrm{CO} \mathrm{Col}_{2} \mathrm{~mol}^{-1}\right)$ and instantaneous water use efficiency (WUE inst; $\mu$ mol $\mathrm{CO}_{2} \mathrm{mmol}^{-1} \mathrm{H}_{2} \mathrm{O}$ ) were also measured on recently expanded leaves of eight plants per treatment by means of a portable photosynthesis (Model LI-6400, LICOR Biosciences Inc., Lincoln, USA). Besides, maximal photochemical efficiency of PSII (Fv/Fm) was assessed at predawn using a handy PEA portable fluorometer (Hansatech, United Kingdom).

\subsection{Determination of electrolyte leakage}

Electrolyte leakage in fresh leaves was determined following Li et al. (2014b) method:

Electrolyte leakage $(\%)=[(\mathrm{EC} 1-\mathrm{EC} 0) /(\mathrm{EC} 2-\mathrm{EC} 0)] \times 100$,

where $\mathrm{EC}_{0}$ is the electrical conductivity recorded at 0 time point; $\mathrm{EC}_{1}$ is the electrical conductivity measured after contents heated at $60^{\circ} \mathrm{C}$; $\mathrm{EC}_{2}$ is the electrical conductivity after contents heated at $100^{\circ} \mathrm{C}$.

\subsection{Determination of ion content}

Concentrations of sodium $\left(\mathrm{Na}^{+}\right)$and potassium $\left(\mathrm{K}^{+}\right)$were determined in oven dried leaf samples, after wet-digesting the samples in $\mathrm{HNO}_{3}-\mathrm{HClO}_{4}$ acid mixture $(4: 1 \mathrm{v} / \mathrm{v})$, by atomic absorption spectrometry (Perkins Elmer, Norwalk, CT, USA) as described by Taïbi et al. (2016).

\subsection{Antioxidant enzymes assay}

Antioxidant enzymes were extracted at $4{ }^{\circ} \mathrm{C}$ from $500 \mathrm{mg}$ fresh leaf sample in Tris- $\mathrm{HCl}$ (100 mM, pH 7.5) containing dithiotrol (5 mM), $\mathrm{MgCl}_{2}(10 \mathrm{mM})$, EDTA $(1.0 \mathrm{mM})$, magnesium acetate $(5 \mathrm{mM})$, PVP (1.5\%), PMSF (1.0 mM) and $1 \mu \mathrm{g} / \mathrm{mL}$ aproptinin in a pre-chilled pestle and mortar. The homogenate was centrifuged at $10.000 \mathrm{rpm}$ for $15 \mathrm{mi}-$ nutes at $4{ }^{\circ} \mathrm{C}$ and the supernatant was used as enzyme source then Superoxide dismutase (SOD), Catalase (CAT), Ascorbate peroxidase (APX) and Glutathione reductase (GR) activity were assayed according to the method described by Cakmak et al., 1993. 


\subsection{Statistical analysis}

Prior to the analysis, the Levene test was applied to check the analysis of variance requirements. The significance of differences between different treatments was assessed using analysis of variance, at $95 \%$ confidence level. The post-hoc Duncun test was used to estimate homogeneous groups. Correlations between the measured parameters were determined based on the coefficient of Pearson. All the statistical analyses were performed using the computing environment $\mathrm{R}$.

\section{Results}

The effect of salt stress on faba bean seedlings growth was evaluated throughout the determination of shoot and root dry weight (Figs. 1A and B). In general, dry biomass decreased significantly in comparison to control seedlings for both genotypes in response to the increase of $\mathrm{NaCl}$ concentration (p-value $<0.01^{* *}$ ). Shoot dry biomass decreased by $60 \%$ in Aguadulce and $65 \%$ in Histal while root dry biomass decreased by around $70 \%$ in both genotypes under high salinity. However, the exogeneous application of salicylic acid enhances both shoot and root biomass over control and salt stressed seedlings; under high salinity, less decrease in shoot dry biomass was observed in the treatment with $0.5 \mathrm{mM}$ SA (50\% for Aguadulce and $60 \%$ for Histal). However, better effect concerning root dry biomass was noted in the treatment with $1 \mathrm{mM}$ SA for both genotypes.

Fig. 1.

As expected, salinity affects seedlings water status; leaf relative water content (RWC) diminished by more than $32 \%$ for both genotypes and water potential $(\Psi \mathrm{w})$ decreased significantly by around $153 \%$ in Aguadulce and $135 \%$ in Histal. This finding indicates that seedlings were experiencing drought stress concomitant to salinity. However, salicylic acid application improves significantly seedlings water status through the decrease of water potential to $165 \%$ for Aguadulce and 150 $\%$ for Histal and therefore, the recovery of relative water content to $72 \%$ and $74 \%$ respectively in Aguadulce and Histal. Nevertheless, under high salinity, better improving effect of water status was observed through treatment with $0.5 \mathrm{mM}$ SA (Fig. 2A, B).

Fig. 2.

Likewise, stomatal conductance (gs), transpiration (E), net photosynthesis $(\mathrm{Pn})$ and intercellular $\mathrm{CO}_{2}$ concentration (Ci) decreased significantly when seedlings were subjected to salt stress (Figs. $3 \mathrm{~A}, \mathrm{~B}, \mathrm{C}$, D). The concomitant diminution under high salinity was around $48 \%$ in gas exchange, $52 \%$ in transpiration and intercellular $\mathrm{CO}_{2}$ concentration, and $34 \%$ in net photosynthesis for both genotypes. Exogenous application of salicylic acid improved significantly these parameters; less decreases were estimated around $34 \%$ and $32 \%$ in gas exchange, $48 \%$ and $53 \%$ for transpiration, $30 \%$ and $31 \%$ in net photosynthesis and 33 $\%$ and $35 \%$ in intercellular concentration of $\mathrm{CO}_{2}$ respectively in Aguadulce and Histal subjected to high salinity. Additionally, maximal efficiency of PSII (Fv/Fm) decreased as well under salinity for both genotypes by around $7 \%$ (p-value $<0.01 \%$ ). Nevertheless, exogeneous salicylic acid enhanced the maximal efficiency of PSII under controlled and salt stressed conditions by around 2 to $3 \%$; the highest value obtained under $200 \mathrm{mM} \mathrm{NaCl}(\mathrm{Fv} / \mathrm{Fm}=0.77)$ was observed in the treatment with $0.5 \mathrm{mM}$ SA for the genotype Aguadulce and in the treatment with $1 \mathrm{mM}$ SA for the genotype Histal (Fig. 3 E).

Subsequent increase in water use efficiency (WUEinst) was induced by salinity and salicylic acid application which improved more the obtained values in both the two genotypes (Fig. 3 F). Nevertheless, water use efficiency was higher in the genotype Aguadulce under controlled and salt-stressed conditions. This improvement translates a higher capacity to maintain elevated photosynthetic levels in the genotype Aguadulce for comparable transpiration values in both genotypes.

Fig. 3.

Being the last sink, leaves constitute the most sensitive plant part where salt ions accumulate. Salt stress induced a significant increase of sodium content by around $156 \%$ against a significant decrease of potassium content by around $50 \%$ for both genotypes (p-value $<0.001^{* * *}$ ). However, exogenous salicylic acid supply decreased remarkably sodium content in leaves of both genotypes by around $10 \%$ for the concentration $0.5 \mathrm{mM} \mathrm{SA}$ and $15 \%$ for the concentration $1 \mathrm{mM}$ SA under high salinity (Fig. 4 A). Concomitantly, potassium content increased for both genotypes by around $30 \%$ under $0.5 \mathrm{mM} \mathrm{SA}$ and $50 \%$ under $1 \mathrm{mM}$ SA when plants were subjected to $200 \mathrm{mM} \mathrm{NaCl}$ (Fig. 4 B). Consequently, the ratio $\mathrm{K}^{+} / \mathrm{Na}^{+}$, which declined under salinity, was improved significantly with salicylic acid treatment (Fig. 4 C).

In addition, salinity affected significantly membrane stability throughout the induced increase of electrolyte leakage by more than four-folds in both genotypes (p-value $<0.001^{* * *}$ ). Treatment of seedlings with salicylic acid diminished widely the electrolyte leakage either in controlled or salt-stressed seedlings. This effect was estimated around $17 \%$ of decrease in Aguadulce and $22 \%$ in Histal under high salinity in comparison to salt-stressed seedlings untreated with SA (Fig. 4 D).

Fig. 4.

Regarding antioxidant enzymes activity, a significant increase was observed for both genotypes subjected to salt stress in terms of superoxide dismutase (SOD), ascorbate peroxidase (APX), catalase (CAT) and glutathione reductase (GR) activities (p-value $<0.001 * * *$ ). The rates of increase were estimated around to $260 \%$ for SOD and APX activity however, CAT activity increased by $210 \%$ in Aguadulce and $224 \%$ in Histal while GR activity increased by $198 \%$ in Aguadulce and $192 \%$ in Histal. The application of salicylic acid induced further increase of the enzymatic activity mainly under high salinity. SOD activity increase was estimated around $300 \%$ for Aguadulce and $320 \%$ for Histal. Nevertheless, APX activity increase was about $280 \%$ for Aguadulce and $270 \%$ for Histal. By the same, CAT activity increased to $260 \%$ for Aguadulce and $270 \%$ for Histal while GR activity raised to $240 \%$ for both genotypes. Better effects were observed in the treatment supplied with $0.5 \mathrm{mM}$ SA for APX, CAT and GR. However, SOD activity was higher in the treatment with $1 \mathrm{mM} \mathrm{SA}$ (Fig. $5 \mathrm{~A}, \mathrm{~B}, \mathrm{C}, \mathrm{D}$ ).

Fig. 5.

\section{Discussion}

Salinity is among the major challenging threats to horticulture by inducing several limitations in plants growth, development and overall productivity. Selection and improvement of salt-tolerant genotypes represents an effective strategy to improve plants productivity in areas
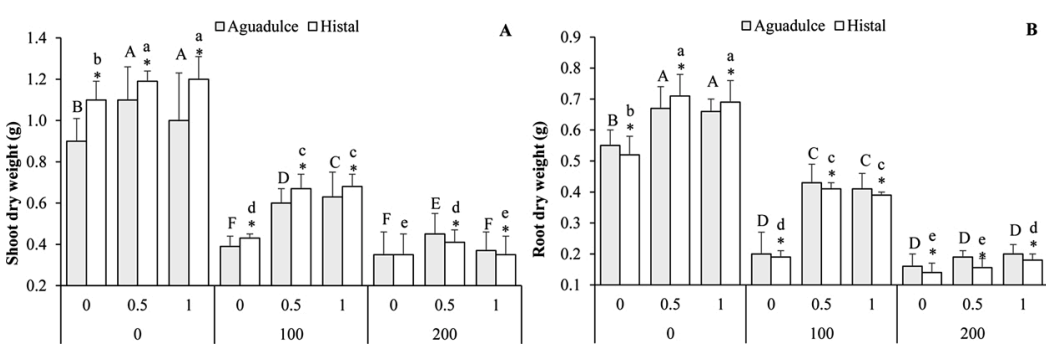

B Fig. 1. Effects of salicylic application on shoot (a) and root (b) dry weight of two faba bean genotypes Aguadulce (grey bars) and Histal (white bars) subjected to salinity.

The letters above the bars marks the significant difference among the different treatments with salt and salicylic acid for each genotype (Capital letters for Aguadulce and small letters for Histal) following the post hoc Duncan's test. The asterisk shows the significant difference between the two genotypes within the same treatment. Scale bars are mean + SE, being the number of samples $n=8$. 

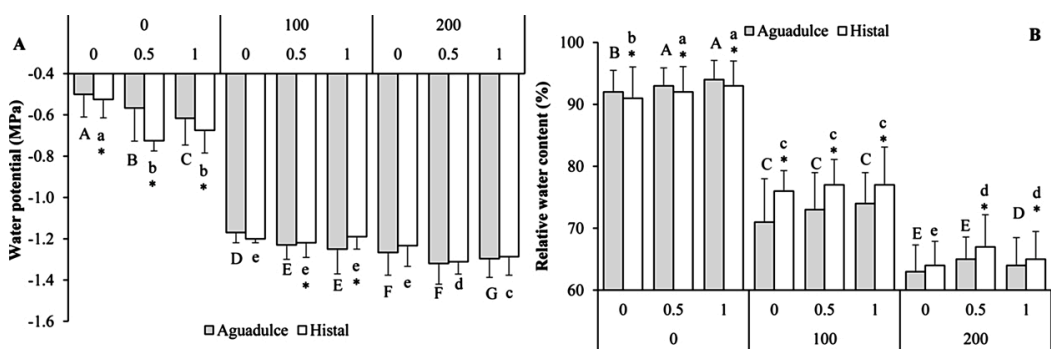
and relative water content (b) of two faba bean genotypes Aguadulce (grey bars) and Histal (white bars) subjected to salinity.

The letters above the bars marks the significant difference among the different treatments with salt and salicylic acid for each genotype (Capital letters for Aguadulce and small letters for Histal) following the post hoc Duncan's test. The asterisk shows the significant difference between the two genotypes within the same treatment. Scale bars are mean $+\mathrm{SE}$, being the number of samples $n=8$.
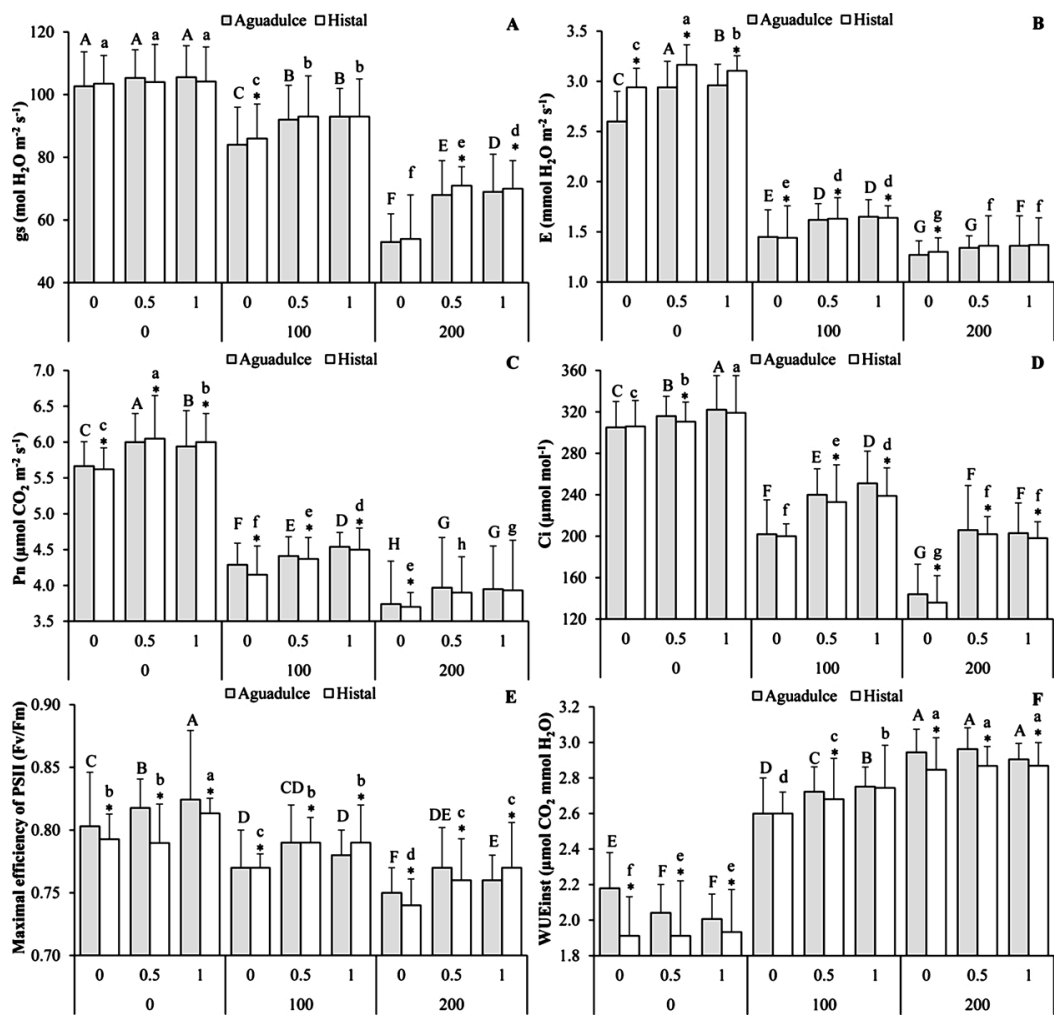

3. Effects of salicylic application on gas exchanges and photosynthesis of two faba bean genotypes Aguadulce (grey bars) and Histal (white bars) subjected to salinity.

Stomatal conductance (gs) (a), transpiration (E) (b), Net photosynthesis $(\mathrm{Pn})(\mathbf{c})$, intercellular $\mathrm{CO}_{2}$ concentration (Ci) (d), Maximal efficiency of PSII (e), and instantaneous water use efficiency (WUEinst) (f).

The letters above the bars marks the significant difference among the different treatments with salt and salicylic acid for each genotype (Capital letters for Aguadulce and small letters for Histal) following the post hoc Duncan's test. The asterisk shows the significant difference between the two genotypes within the same treatment. Scale bars are mean $+\mathrm{SE}$, being the number of samples $n=8$.
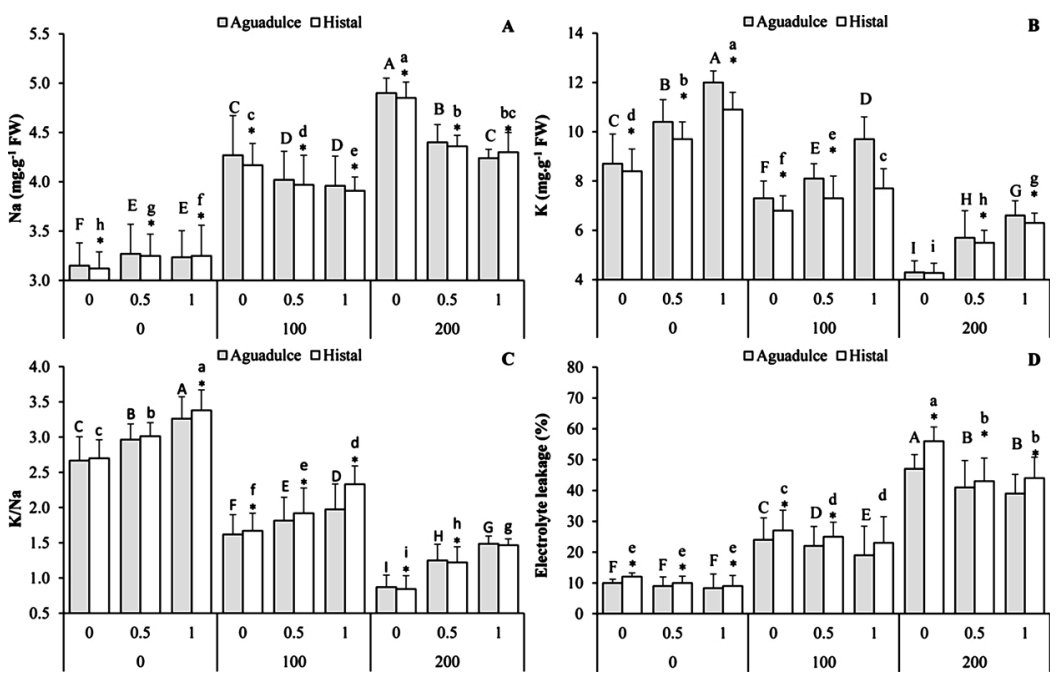

B and Histal (white bars) subjected to salinity.

Sodium content $\left(\mathrm{Na}^{+}\right)$(a), potassium content $\left(\mathrm{K}^{+}\right)(\mathbf{b})$, ratio $\mathrm{K}^{+}$/ $\mathrm{Na}^{+}$(c) and electrolyte leakage (d).

The letters above the bars marks the significant difference among the different treatments with salt and salicylic acid for each genotype (Capital letters for Aguadulce and small letters for Histal) following the post hoc Duncan's test. The asterisk shows the significant difference between the two genotypes within the same treatment. Scale bars are mean + SE, being the number of samples $n=8$. 

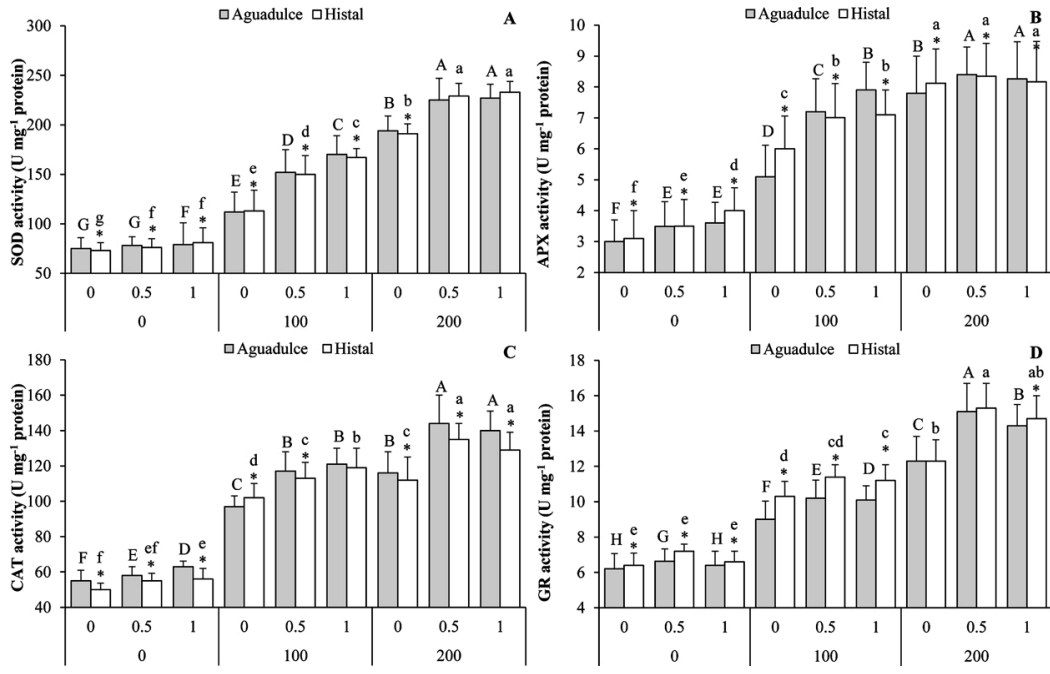

a $\begin{gathered}\text { D } \\ \text { ab }\end{gathered}$ $n=8$

Fig. 5. Effects of salicylic application on antioxidant enzymes activities of two faba bean genotypes Aguadulce (grey bars) and Histal (white bars) subjected to salinity.

Superoxide dismutase activity (SOD) (a), Ascorbate peroxidase activity (APX) (b), Catalase activity (CAT) (c) and Glutathione reductase activity (GR) (d).

The letters above the bars marks the significant difference among the different treatments with salt and salicylic acid for each genotype (Capital letters for Aguadulce and small letters for Histal) following the post hoc Duncan's test. The asterisk shows the significant difference between the two genotypes within the same treatment. Scale bars are mean + SE, being the number of samples $n=8$.

suffering from adverse environmental conditions (Hernández 2019). Therefore, comparison among genotypes for salt tolerance can be very useful to describe the relevant mechanisms that might help to recognize stress tolerant genotypes and reveal the limiting factors which could be used for molecular breeding (Hanin et al., 2016; Taïbi et al., 2017; Taïbi et al., 2018). Besides, phytohormones constitute a strong tool to alleviate the adverse effects produced by abiotic stress. Recently, an increasing interest has been focused on the effect of salicylic acid in salinity tolerance (Khan et al. 2015).

In general, salinity induced severe restrictions of physiological and molecular responses of faba bean which affected significantly plants growth. However, salicylic acid restored most of these parameters and improve the plants stress response.

Severe growth limitations in terms of shoot and root dry biomass were observed under salinity for both genotypes. This finding, as one of the most visible effects of salt stress, is generally attributed to the inhibition of the developmental program and the stimulation of stress response mechanisms (Isayenkov and Maathuis, 2019). Growth arrest under salinity has been already reported in faba bean (Bulut and Eroğlu 2011; Dawood and El-Awadi, 2015; Moussa and Hassan, 2016) and other leguminous species like common bean (Taïbi et al., 2012a, b; Taïbi et al., 2013a, b; Taïbi et al., 2016). Therefore, biomass accumulation measure may help in the evaluation of the relative degree of salt tolerance among faba bean genotypes. However, the application of salicylic acid enhanced both shoot and root dry biomass in salt-stressed plants. The ameliorating effects of salicylic acid could be attributed to its role in membrane protection from salt damage ( $r$-value $=0.73 * *$ ), and enhancement of chlorophyll content and antioxidant enzymes activity (r-value $\left.=0.82^{* *}\right)$ which in turn supports the photosynthesis process, alleviate oxidative stress and regulate ion homeostasis (Li et al., 2014a; Li et al., 2014b; Khan et al., 2015). On the other side, growth limitations could be due to the significant deterioration of relative water content (RWC, r-value $\left.=-0.68^{* *}\right)$ and water potential $(\Psi \mathrm{w}, \mathrm{r}$-value $=-0.71 * *)$ under salinity. It is well admitted that plants including faba bean respond to salt stress by regulating their osmotic potential and ionic balance throughout the accumulation of organic and inorganic compounds in order to sustain cell turgor and subsequently to maintain growth (Taibi and Chorfi, 2010; Abdul Qados, 2011; Chorfi and Taibi, 2011a, b; Nazar et al., 2015). Maintenance of water status through adjustment of water potential is considered as an important adaptation strategy under salinity (Ashraf and Harris, 2013). Application of salicylic acid enhanced leaves diffusive resistance $\left(r\right.$-value $\left.=0.58^{* *}\right)$ and control transpiration level ( $r$-value $=-0.54 * *)$ which might improve water status of the plants (Ahmad et al., 2018).

Likewise, gas exchanges and net photosynthesis decreased in plants subjected to salinity ( $r$-value $=-0.44 *$ ). The stomatal closure, as an earliest response of salt-stressed plants, was significantly marked in the present study leading to the decrease of stomatal conductance and transpiration. In general, higher stomatal conductance enhances $\mathrm{CO}_{2}$ diffusion into the leaves and improves photosynthetic rate which is an important determinant of growth and yield (Li et al., 2014b). Therefore, the decrease in photosynthesis rates under saline conditions is attributed mostly to the limitation of stomatal conductance ( $r$-value $\left.=0.61^{* *}\right)$. Besides, diminution in photosynthesis might be attributed to perturbations in pigments content and chlorophyll fluorescence, PSII photoinhibition, conformational modifications in membrane-bound ATPase enzyme complex, and the diminution in Rubisco activity (Lawlor and Cornic, 2002). Nevertheless, salicylic acid was found to enhance photosynthetic rates and gas exchanges. Previous studies of Khan et al. (2014) and Ahmad et al. (2018) have reported that salicylic acid increases pigments content, improves the rates of transpiration and photosynthetic electron transport, sustains higher Rubisco and antioxidant enzymatic activities, and boosts the PSII efficiency under salt stress. The maximal quantum yield of PSII phytochemistry (Fv/Fm) decreased under saline conditions but restored after the addition of salicylic acid. This finding indicates reduced leaves capacity for absorption of excitation energy and diminution in photosynthetic assimilation under salinity (Taïbi et al., 2017; Taïbi et al., 2018). The protective effects of salicylic acid could be associated to the diminution of oxidative damage and the restriction of massive sodium accumulation in leaves (Khan et al., 2015).

By the same, regulation of ion homeostasis is one of the main determinants of plants salt tolerance (Isayenkov and Maathuis, 2019). In the present study, salinity increased the content of sodium and decreased the content of potassium. These findings are in agreement with (Bulut et al., 2011; Taïbi et al., 2016; Ahmad et al., 2018). Competition exists for the uptake of sodium and potassium since they share similar physicochemical structure. Under salt conditions, the passive entrance of sodium ions under salinity throughout the plasma membrane could induce membrane depolarization that prevents considerably potassium uptake and increases its leakage through depolarization-activated potassium outward-rectifying channels (Jayakannan et al., 2013). The suppression of potassium uptake induces various cell damages and reduces plant growth since it constitutes an important activator of several cytosolic enzymes and contributes as well for osmotic adjustment (Almeida et al., 2017). Besides, potassium plays a determinant role in regulating stomatal aperture, so the observed differences may be the underlying cause for the differences observed in water use efficiency and photosynthesis rates. Exogenous salicylic acid supply was found to be very effective in dropping sodium excess and enhancing potassium content in salt-stressed faba bean plants. Salicylic 
acid reduces the xylemic translocation of sodium to the aerial parts from a part, and the extent of membrane polarization through the stimulation of $\mathrm{H}^{+}$-ATPase activity under salt conditions from another part which might consequently restore potassium retention and decrease its efflux (Khan et al., 2015). Nevertheless, the higher accumulations of sodium and potassium were characteristics of the genotype Aguadulce. This suggests that it is the ability to uptake and retain potassium that determines faba bean genotypes growth and yield under salt stress.

In addition, an increase of electrolyte leakage was associated to salt stress reflecting consequently the occurrence of membrane injuries. Membrane disorganization is due mainly to the decrease of phospholipids and sterols in response to salinity (Wu et al., 1998). Reactive oxygen species attack linolenic and linoleic polyunsaturated fatty acids and produce complex of lipid hydroxy-peroxides which reduce membrane permeability (Ahmad et al., 2018). It should be noted that membrane protection from salt injuries constitutes a determinant criterion for salinity tolerance mechanisms and salicylic acid demonstrated its benefits to decrease electrolyte leakage. Salicylic acid effects are attributed to the stimulation of the antioxidative system and improvement of calcium uptake (El-Tayeb, 2005).

Salt stress affects various physiological and molecular mechanisms associated with plant growth and development. Plants with potent antioxidant system, either constitutive or induced, acquire significant salt-tolerance (Isayenkov and Maathuis, 2019). The activity of the antioxidant enzymes SOD, APX, CAT and GR increased in response to salt stress. The genotype Aguadulce revealed higher SOD and CAT activities while the genotype Histal showed higher APX and GR activities. The role of antioxidant enzymes is to scavenge reactive oxygen species produced by oxidative stress and therefore protect cell membrane integrity (Taïbi et al., 2016). SOD converts superoxide radicals into which will be reduced by CAT to $\mathrm{H}_{2} \mathrm{O}$ and oxygen (Demiral and Türkan, 2005). Besides, the increase in GR activity under salt conditions has been also reported (Maribel and Tobita, 1998; Taïbi et al., 2016). The antioxidant enzymatic activities increased in controlled and salt-stressed conditions after salicylic acid treatments. Exogenous application of salicylic acid mitigated salt stress in S. lycopersicum throughout the stimulation of GST gene family (Csiszár et al. 2014). The boosted expression of antioxidants genes such as GST1-2, GPX1-2 and ASA-GSH pathway in response to salicylic acid supply restores cell membrane integrity and enhances the content of photosynthetic pigments (Li et al., $2014 \mathrm{~b}$ ). It should be noted that the effect of salicylic acid varied according to the applied concentration; CAT, APX and GR activities were stimulated the maximum at $0.5 \mathrm{mM}$ SA under high salinity while SOD activity was stimulated at $1 \mathrm{mM}$ SA.

Taken all together, even if the genotype Aguadulce performed better than the genotype Histal in terms of growth, physiological and molecular response under salt stress, the difference between genotypes remains more quantitative rather than qualitative. Salicylic acid application improved significantly the salt-tolerance of both the two genotypes throughout the maintenance of cell membrane and photosynthetic process and the diminution of sodium toxicity and oxidative damages. Nevertheless, the action of salicylic acid varies in function of the applied concentration of the hormone, the tested genotype and the studied parameter. Overall, the genotype Aguadulce performs better with the treatment $0.5 \mathrm{mM}$ SA while the genotype Histal manifests greater behaviour with the treatment $1 \mathrm{mM}$ SA.

\section{Conclusion}

The intraspecific comparative studies among genotypes for salttolerance might help to identify the relevant mechanisms correlating their relative degree of tolerance to salinity and to select the most suited genotypes for very specific environmental conditions.

The present study revealed that the difference between genotypes is more quantitative rather than qualitative even if the genotype Aguadulce performed better in terms of growth, physiological and molecular response under salt stress. In general, the salt stress promoted several limitations in growth, gas exchanges, photosynthesis, ion homeostasis and other physiological attributes. Nevertheless, faba bean plants have triggered some mechanisms to deal with this constraint throughout the maintenance of water status and the enhancement of antioxidant enzymatic activities. However, the salt-tolerance of both the two genotypes was significantly improved by salicylic acid application which allowed the maintenance of cell membrane and photosynthetic process, restoring of ion homeostasis and the diminution of oxidative damages. The beneficial effects of salicylic acid vary according to its concentration, the tested genotype and the studied parameter. Overall, the genotype Aguadulce performs better under the treatment with $0.5 \mathrm{mM} \mathrm{SA}$ while the genotype Histal manifests greater behaviour under the treatment with $1 \mathrm{mM}$ SA.

Based on our results, salicylic acid can be considered as potential growth regulator to improve the salt response of faba bean. Plants treatment with salicylic acid might constitute a sustainable approach and effective alternative to restore the adverse effects of salinity. The application of salicylic acid would provide a practical basis for wide cultivation of faba bean and might propose an ecological and economical solution to deal with salt-affected soils, mainly in arid regions, for the reclamation of marginal and wastelands under-cultivated.

\section{CRediT authorship contribution statement}

Kada Souana: Methodology, Validation, Formal analysis, Investigation, Writing-Original Draft, Visualization. Khaled Taïbi: Conceptualisation, Methodology, Validation, Formal analysis, Investigation, Writing-Original Draft, Writing-Review \& Editing, Resources, Visualization, Supervision, Project administration. Leila Ait Abderrahim: Methodology, Investigation, Writing-Original Draft, Writing-Review \& Editing, Visualization. Mokhtar Amirat: Investigation, Validation, Visualization. Mohamed Achir: Investigation, Validation, Visualization. Mohamed Boussaid: Resources, Visualization, Funding acquisition. José M. Mulet: Resources, Visualization, Funding acquisition.

\section{Declaration of Competing Interest}

The authors report no declarations of interest.

\section{Appendix A. Supplementary data}

Supplementary material related to this article can be found, in the online version, at https://doi.org/10.1016/j.scienta.2020.109641.

\section{References}

Abdul Qados, A.M.S., 2011. Effect of salt stress on plant growth and metabolism of bean plant Vicia faba (L.). J. Saudi Soc. Agric. Sci. 10, 7-15.

Ahmad, P., Alyemeni, M.N., Ahanger, M.A., Egamberdieva, D., Wijaya, L., Alam, P., 2018. Salicylic acid (SA) induced alterations in growth, biochemical attributes and antioxidant enzyme activity in Faba bean (Vicia faba L.) seedlings under $\mathrm{NaCl}$ toxicity. Russ. J. Plant. Physl. 65, 104-114.

Almeida, D.M., Oliveira, M.M., Saibo, N.J.M., 2017. Regulation of $\mathrm{Na}^{+}$and $\mathrm{K}^{+}$ homeostasis in plants: towards improved salt stress tolerance in crop plants. Genet. Mol. Biol. 40, 326-345.

Ashraf, M., Harris, P.J.C., 2013. Photosynthesis under stressful environments: An overview. Photosynthetica. 51, 163-190.

Bulut, F., Akıncı, S.., Eroğlu, A., 2011. Growth and uptake of sodium and potassium in Broad bean (Vicia faba L.) under salinity stress. Commun. Soil. Sci. Plan. 42, 945-961.

Cakmak, I., Strbac, D., Marschner, H., 1993. Activities of hydro-gen peroxide scavenging enzymes in germinated wheat seeds. J. Exp. Bot. 44, 127-132.

Castro-Guerrero, N.A., Cui, Y., Mendoza-Cozatl, D., 2016. Purification of translating ribosomes and associated mRNAs from soybean (Glycine max). Curr. Protoc. Plant Biol . 1, 1-12. https://doi.org/10.1002/cppb.20011.

Chorfi, A., Taibi, K., 2011a. Physiological behavior of wheat genotypes from Algerian semi-arid regions grown under salt stress. Afr. J. Agric. Res. 6 (3), 636-641.

Chorfi, A., Taïbi, K., 2011b. Biochemical screening for osmotic adjustment of wheat genotypes under drought stress. Tropicultura 29 (2), 82-87.

Csiszár, J., Horváth, E., Váry, Z., Gallé, Á., Bela, K., Brunner, S., Tari, I., 2014. Glutathione transferase supergene family in tomato: Salt stress-regulated expression 
of representative genes from distinct GST classes in plants primed with salicylic acid. Plant. Physiol. Biochem. 78, 15-26.

Dawood, M.G., El-Awadi, M.E., 2015. Alleviation of salinity stress on Vicia faba L. plants via seed priming with Melatonin. Acta. Biol. Colomb. 20, 223-235.

Demiral, T., Türkan, I., 2005. Comparative lipid peroxidation, antioxidant defense systems and proline content in roots of two rice cultivars differing in salt tolerance. Environ. Exp. Bot. 53, 247-257.

El-Tayeb, M.A., 2005. Response of barley grains to the interactive effect of salinity and salicylic acid. Plant. Growth. Regul. 45, 215-224.

Hanin, M., Ebel, C., Ngom, M., Laplaze, L., Masmoudi, K., 2016. New insights on plant salt tolerance mechanisms and their potential use for breeding. Front. Plant Sci. 7, 1787. https://doi.org/10.3389/fpls.2016.01787.

Hernández, J.A., 2019. Salinity tolerance in plants: trends and perspectives. Int. J. Mol. Sci. 20 (10), 2408. https://doi.org/10.3390/ijms20102408.

Herrera-Vásquez, A., Salinas, P., Holuigue, L., 2015. Salicylic acid and reactive oxygen species interplay in the transcriptional control of defense genes expression. Front. Plant. Sci. 6, 171. https://doi.org/10.3389/fpls.2015.00171.

Isayenkov, S.V., Maathuis, F.J.M., 2019. Plant salinity stress: many unanswered questions remain. Front. Plant. Sci. 10, 80. https://doi.org/10.3389/ fpls.2019.00080.

Jayakannan, M., Bose, J., Babourina, O., Rengel, Z., Shabala, S., 2013. Salicylic acid improves salinity tolerance in Arabidopsis by restoring membrane potential and preventing salt-induced $\mathrm{K}^{+}$loss via a GORK channel. J. Exp. Bot. 64, 2255-2268.

Khan, M.I.R., Asgher, M., Khan, N.A., 2014. Alleviation of salt-induced photosynthesis and growth inhibition by salicylic acid involves glycinebetaine and ethylene in mungbean (Vigna radiata L.). Plant. Physiol. Biochem. 80, 67-74.

Khan, M.I.R., Fatma, M., Per, T.S., Anjum, N.A., Khan, N.A., 2015. Salicylic acid-induced abiotic stress tolerance and underlying mechanisms in plants. Front. Plant. Sci. 6, 462. https://doi.org/10.3389/fpls.2015.00462.

Lawlor, D.W., Cornic, G., 2002. Photosynthetic carbon assimilation and associated metabolism in relation to water deficits in higher plants. Plant. cell. environ. 25, 275-294.

Li, L., Zhang, H., Zhang, L., Zhou, Y., Yang, R., Ding, C., Wang, X., 2014a. The physiological response of Artemisia annua L. to salt stress and salicylic acid treatment. Physiol. Mol. Biol. Plants. 20, 161-169.

Li, T., Hu, Y., Du, X., Tang, H., Shen, C., Wu, J., 2014b. Salicylic acid alleviates the adverse effects of salt stress in Torreya grandis cv. Merrillii seedlings by activating photosynthesis and enhancing antioxidant systems. PLOS ONE 9, e109492.
Maribel, D.-S., Tobita, S., 1998. Antioxidant responses of rice seedlings to salinity stress. Plant. Sci. 135, 1-9.

Moussa, H.R., Hassan, M.A.E.-F., 2016. Growth enhancers to mitigate salinity stress in Vicia faba. Intern. J. Veget. Sci. 22, 243-250.

Nazar, R., Umar, S., Khan, N.A., 2015. Exogenous salicylic acid improves photosynthesis and growth through increase in ascorbate-glutathione metabolism and S assimilation in mustard under salt stress. Plant. Signal. Behav. 10, e1003751.

Taïbi, K., Boussaid, M., Achir, M., Taïbi, F., Belkhodja, M., 2012b. Evaluation of growth response and water relations of two bean genotypes (Phaseolus vulgaris L.) from Algerian semi-arid regions grown under salt stress. Genetics. Plant. Physiol. 3-4, $176-185$.

Taïbi, K., Chorfi, A., 2010. Physiological behavior of two Algerian wheat genotypes grown under saline conditions. Arab. Gulf. J. Sci. Res. 28 (4), 197-204.

Taïbi, K., Del Campo, A.D., Vilagrosa, A., Bellés, J.M., López-Gresa, M.P., LópezNicolás, J.M., Mulet, J.M., 2018. Distinctive physiological and molecular responses to cold stress among cold-tolerant and cold-sensitive Pinus halepensis seed sources. BMC Plant Biol. 18, 236.

Taïbi, K., del Campo, A.D., Vilagrosa, A., Bellés, J.M., López-Gresa, M.P., Pla, D., Calvete, J.J., López-Nicolás, J.M., Mulet, J.M., 2017. Drought tolerance in Pinus halepensis seed sources as identified by distinctive physiological and molecular markers. Front. Plant. Sci. 8, 1202. https://doi.org/10.3389/fpls.2017.01202.

Taïbi, K., Taïbi, F., Ait Abderrahim, L., Ennajah, A., Belkhodja, M., Mulet, J.M., 2016. Effect of salt stress on growth, chlorophyll content, lipid peroxidation and antioxidant defence systems in Phaseolus vulgaris L. S. Afr. J. Bot. 105, 306-312.

Taïbi, K., Taïbi, F., Belkhodja, M., 2012a. Effects of external calcium supply on the physiological response of salt stressed bean (Phaseolus vulgaris L.). Genetics. Plant. Physiol. 2, 177-186.

Taïbi, K., Taïbi, F., Belkhodja, M., 2013a. Plants growth, water relations and photosynthesis of two bean genotypes Phaseolus vulgaris L. treated with $\mathrm{NaCl}$ and fluridone. Afric. J. Biotech. 12, 3811-3821.

Taïbi, K., Taïbi, F., Belkhodja, M., 2013b. Salinity Effects on the physiological response of two bean genotypes (Phaseolus vulgaris L.). Arab. Gulf. J. Sci. Res. 31, 90-98.

Wu, J., Seliskar, D.M., Gallagher, J.L., 1998. Stress tolerance in the marsh plant Spartina patens: Impact of $\mathrm{NaCl}$ on growth and root plasma membrane lipid composition. Physiol. Plant. 102, 307-317.

Xu, E., Brosché, M., 2014. Salicylic acid signaling inhibits apoplastic reactive oxygen species signaling. BMC Plant. Biol. 14, 155. 